\title{
Applications of Automated High Resolution Strain Mapping in TEM on the Study of Strain Distribution in MOSFETs
}

\author{
A. D. Darbal ${ }^{1,4}$, R. D. Narayan ${ }^{1}$, C. Vartuli ${ }^{2}$, T. Aoki ${ }^{3}$, J. Mardinly $^{3}$, S. Nicolopoulos ${ }^{4}$ and J. K. Weiss ${ }^{1}$ \\ 1. AppFive LLC, Tempe, USA. \\ 2. Texas Instruments, Dallas, USA. \\ 3. LeRoy Eyring Center for Solid State Science, Arizona State University, Tempe, USA. \\ 4. NanoMEGAS SPRL, Brussels, Belgium
}

Engineered strain in the channel is a cost effective means of improving the performance of devices [1]. Through deliberately introducing strain in silicon, the band gap is altered, which in turn can be used to enhance the electron mobility. A metrology tool for reliable channel strain determination is crucial for process development. Given the scale of modern semiconductor devices, electron diffraction in the transmission electron microscope (TEM) is well suited for this purpose. In particular, nanobeam diffraction (NBD) is interesting as it is possible to acquire NBD patterns at high spatial resolution and is relatively easy to set up in most modern TEMs [2]. However, the presence of strong dynamical effects in conventional NBD patterns makes it difficult to obtain reliable strain data as the spot intensity distributions are strongly dependent on local specimen thickness and orientation. This is overcome by precessing the beam as the diffraction patterns are acquired [3]. With precession the incident beam is tilted and rotated (typically at $100 \mathrm{~Hz}$ ) about the optic axis so that the dynamical effects are averaged out and the diffraction patterns are acquired under quasi-kinematical conditions. In addition, precession electron diffraction (PED) patterns have better sampling of higher order reflections, which are more sensitive to small changes in lattice parameters.

In an earlier work, we had demonstrated the use of PED patterns for high precision strain mapping in the TEM [4]. In this technique, PED spot patterns are acquired in the TEM under nanobeam illumination and are compared with a reference pattern from the unstrained region of the same material. The in-plane strain tensor is determined by measuring the distortion in the strain pattern by fitting the entire strain pattern to the unstrained reference pattern. Fitting the entire pattern leads to improvement in strain precision and is amenable for a high degree of automation.

In this work, we apply the aforementioned technique to the strain mapping of a pMOS and an nMOS field effect transistors (FET). A JEOL ARM 200F, operated at $200 \mathrm{kV}$, was used for this study. A precession angle of $0.7^{\circ}$ was used to acquire the patterns. Topspin system, developed by AppFive LLC, was used for synchronized beam scanning with precession. The nMOS strain maps were acquired at 2 $\mathrm{nm}$ step size with $200 \times 65$ points and the pMOS strain maps were acquired at $3 \mathrm{~nm}$ with $200 \times 82$ points. The total acquisition and analysis for each of the maps is under 5 minutes. Figs. 1 and 2 show the results for the nMOS and pMOS samples respectively. Using the Topspin system, we also acquire reference scanning TEM (STEM) bright field images. The insets in the reference images in Figs. 1 and 2 show reconstructed bright field images using the diffraction patterns. The reconstructed bright field is useful to identify the exact area scanned.

From the strain maps, we observe a tensile strain of $0.8 \%$ and a compressive strain of $0.5 \%$ along the direction of electron flow for the nMOS and pMOS samples, respectively. These results were further corroborated with complementary techniques. 


\section{References:}

[1] PR Chidambaram, et al., IEEE Transactions on Electron Devices, 53 5, (2006).

[2] D Cooper et al., Journal of Physics: Conference Series 326012025 (2011), p.944 -964.

[3] R Vincent, PA Midgley, Ultramicroscopy 53 (1994) p. 271.

[4] Darbal et al., Proceedings of Microscopy and Microanalysis, 19, S2, (2013), pp. 702-703.

[5] We gratefully acknowledge the use of facilities within the LeRoy Eyring Center for Solid State Science at Arizona State University.
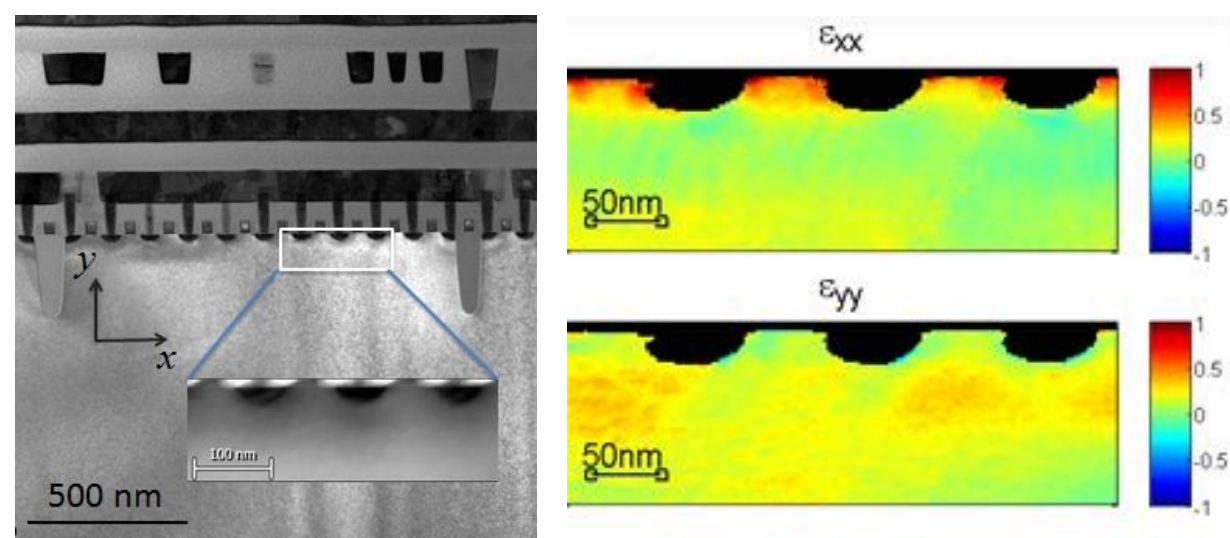

Figure 1. STEM reference image and the virtual bright field image (left panel), and the corresponding strain maps for the nMOS sample (right panel).
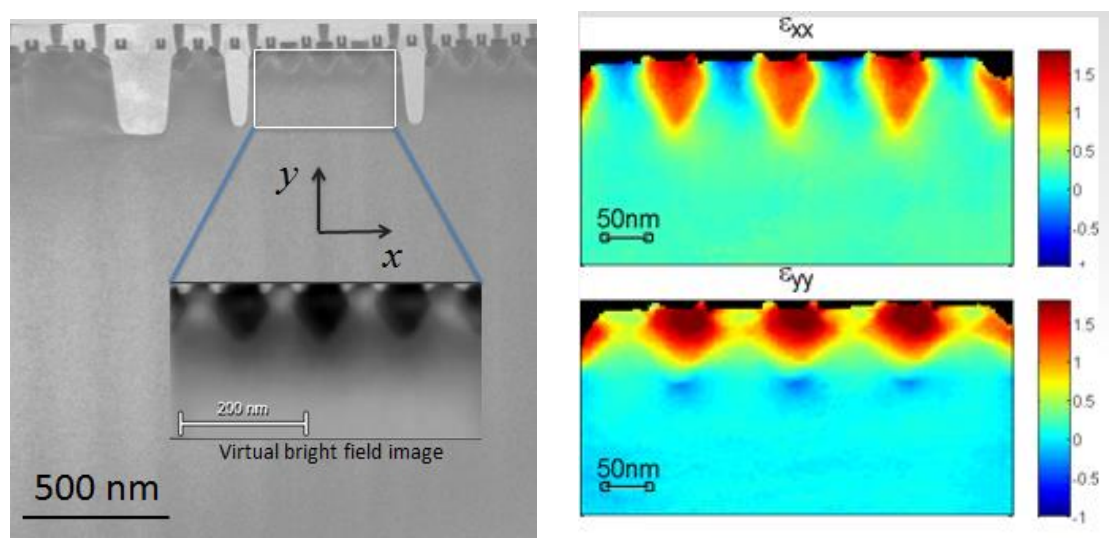

Figure 2. STEM reference image and the virtual bright field image (left panel), and the corresponding strain maps for the pMOS sample (right panel). 